\title{
Optical Pump - Terahertz Probe Measurement of the Electron Dynamics in $\mathrm{Ga}_{1-x} \mathrm{Mn}_{x} \mathrm{As}$
}

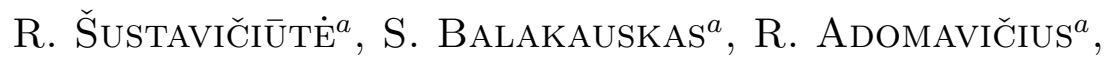 \\ A. KRotKus ${ }^{a}$ AND J. SADOWSKI ${ }^{b}$ \\ ${ }^{a}$ Semiconductor Physics Institute \\ A. Gostauto 11, 01108 Vilnius, Lithuania \\ ${ }^{b}$ Institute of Physics, Polish Academy of Sciences \\ al. Lotników 32/46, 02-668 Warsaw, Poland
}

\begin{abstract}
An optical pump - terahertz probe technique was used for measuring electron lifetime in various $\mathrm{Ga}_{1-x} \mathrm{Mn}_{x}$ As epitaxial layers with the subpicosecond temporal resolution. The measurements were performed on the samples with $x$ up to $2 \%$, which had large resistivities and were transparent in a $\mathrm{THz}$ frequency range. It has been found that an induced $\mathrm{THz}$ absorption relaxation is the fastest and electron lifetimes are the shortest for the samples with the smallest Mn content. For the samples with $x=0.3 \%$ and $x=2 \%$ this relaxation becomes much slower; its rate is comparable to the carrier recombination rate in $\mathrm{Ga}_{1-x} \mathrm{Mn}_{x}$ As substrate.
\end{abstract}

PACS numbers: 42.65.Re, 07.57.Hm, 78.47.+p, 75.50.Pp

\section{Introduction}

$\mathrm{Ga}_{1-x} \mathrm{Mn}_{x}$ As and related alloys are promising candidates for spintronic devices compatible with the conventional GaAs technology. The Curie temperatures as large as $173 \mathrm{~K}$ have been obtained for this material [1]. The ferromagnetism of $\mathrm{Ga}_{1-x} \mathrm{Mn}_{x}$ As is caused not by a direct magnetic exchange interaction between these atoms but is mediated by delocalized current carriers. It is recognized that free holes are responsible for the ferromagnetic interaction in this alloy [2]. Usually a hole density in $\mathrm{Ga}_{1-x} \mathrm{Mn}_{x}$ As layers is significantly smaller than the concentration of $\mathrm{Mn}$ atoms, which is attributed to a large number of defects that create donor centers compensating $\mathrm{Mn}^{++}$acceptors. Those are mainly arsenic antisites $\left(\mathrm{As}_{\mathrm{Ga}}\right)$ arising due to the low-temperature molecular-beam-epitaxy growth of GaAs (LTG 
GaAs) and manganese interstitials $\left(\mathrm{Mn}_{\mathrm{i}}\right)$ that, similarly to the As $\mathrm{Ga}_{\mathrm{Ga}}$ defects, are double-charged donors.

In LTG GaAs $\mathrm{As}_{\mathrm{Ga}}$ are known as very efficient electron recombination centers that cause extremely short, subpicosecond carrier lifetimes in this material [3]. For a spin-injector material such short carrier lifetimes could have a deteriorating effect, therefore an investigation of the non-equilibrium electron dynamics in $\mathrm{Ga}_{1-x} \mathrm{Mn}_{x} \mathrm{As}$ is of a great importance. In the case of LTG GaAs and related semiconductors the electron lifetime is determined by using an optical pump - terahertz probe technique [4], which allows the monitoring of the photoexcited electron density dynamics with subpicosecond temporal resolution. In the present work, we have used this technique for investigating electron lifetimes in $\mathrm{Ga}_{1-x} \mathrm{Mn}_{x} \mathrm{As}$ alloys with widely varying compositions $(0.01 \%<x<2 \%)$. The shortest electron lifetimes $(\approx 1 \mathrm{ps})$ were evidenced for the alloys with the lowest Mn content; in the alloys with more than $0.3 \% \mathrm{Mn}$ the lifetime has increased to more than $100 \mathrm{ps}$.

\section{Experimental}

We investigated $\mathrm{Ga}_{1-x} \mathrm{Mn}_{x}$ As layers grown on semi-insulating (100) GaAs substrates with LTG GaAs buffer layers. Mn content in the layers was increased from $0.01 \%$ to $4.5 \%$. The samples were grown in Kryovak MBE system dedicated to III-Mn-V magnetic semiconductor growth. A valved cracker source was used to generate $\mathrm{As}_{2}$ flux. Before the growth of $\mathrm{Ga}_{1-x} \mathrm{Mn}_{x}$ As layers, the LTG GaAs buffers were deposited at the same substrate temperature $\left(240^{\circ} \mathrm{C}\right)$ and at the same growth rate $\left(0.2\right.$ monolayers/s) as it was further used for $\mathrm{Ga}_{1-x} \mathrm{Mn}_{x}$ As growth. The $\mathrm{As}_{2} / \mathrm{Ga}$ flux ratio was constant and equal to 2 for all growth runs. The Mn content was set by the temperature of the Mn effusion cell and verified by measuring the differences in the growth rates between $\mathrm{Ga}_{1-x} \mathrm{Mn}_{x}$ As and LTG GaAs using reflection high-energy electron diffraction intensity oscillations [5].

Photoexcited carrier relaxation in $\mathrm{Ga}_{1-x} \mathrm{Mn}_{x}$ As layers has been studied by the optical pump - terahertz probe technique. Ti:sapphire laser pulses (150 fs, $76 \mathrm{MHz}, 815 \mathrm{~nm}$ ) were employed in this experiment. $350 \mu \mathrm{m}$ diameter pinhole was used to overlap $\mathrm{THz}$ and visible beams on the sample. The spot size of the pump beam was larger than the diameter of the pinhole so that the $\mathrm{THz}$ probe beam was sampling a nearly uniformly photoexcited region. To maximize the transmitted power through the pinhole, the $\mathrm{THz}$ beam was focused before and collimated after the pinhole by two hemispherical Teflon lenses. THz transients were generated and detected by a pair of photoconductive antennae from LTG GaAs. Because $\mathrm{THz}$ pulses were generated at the laser illuminated $\mathrm{Ga}_{1-x} \mathrm{Mn}_{x} \mathrm{As}$ surface itself, the $\mathrm{THz}$ transmittivity transient was obtained by subtracting this pulse from the measured optical pump - terahertz probe trace. The temporal resolution of the measurement was $\approx 0.7 \mathrm{ps}$; carrier lifetimes were measured when an average power of the optical pump beam was changed from $4 \mathrm{~mW}$ to $260 \mathrm{~mW}$, which corresponded to the photoexcited electron and hole densities in the range from $4 \times 10^{15} \mathrm{~cm}^{-3}$ to $2 \times 10^{17} \mathrm{~cm}^{-3}$. 


\section{Results and discussion}

Figure 1 shows optically induced $\mathrm{THz}$ transmission transients measured on several $\mathrm{Ga}_{1-x} \mathrm{Mn}_{x}$ As samples with different Mn contents. The measurements were performed on the samples with $x$ up to $2 \%$, which had large resistivities and were transparent in the $\mathrm{THz}$ frequency range. The sample with $x=4.5 \%$ was not measured because of the low transmission. As it can be seen from this figure, the induced $\mathrm{THz}$ absorption relaxation is the fastest and electron lifetimes are the shortest for the samples with the smallest Mn content of several hundredths of percent. For the samples with $x=0.3 \%$ and $x=2 \%$ this relaxation becomes much slower; its rate was comparable to the carrier recombination rate in GaAs substrate.

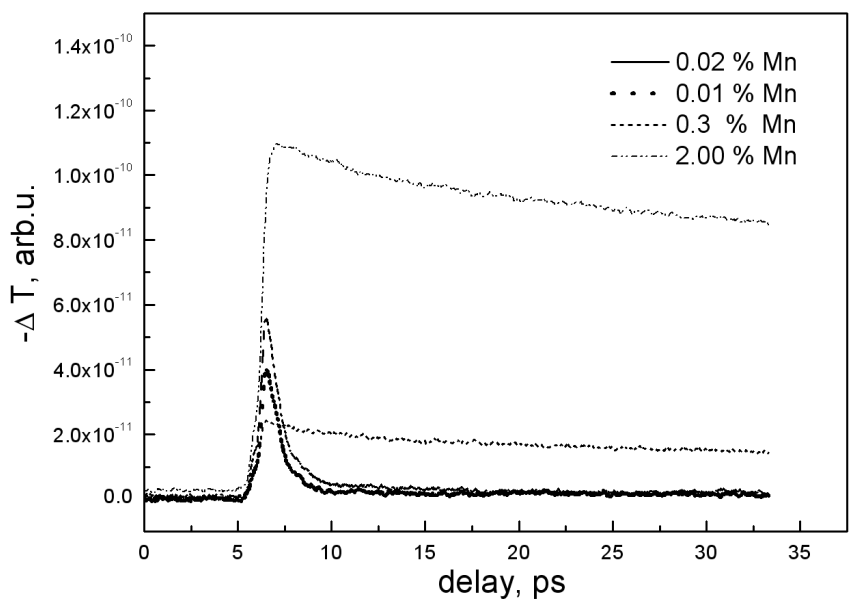

Fig. 1. Optically induced $\mathrm{THz}$ transmission transients measured on several $\mathrm{Ga}_{1-x} \mathrm{Mn}_{x}$ As samples with different Mn contents.

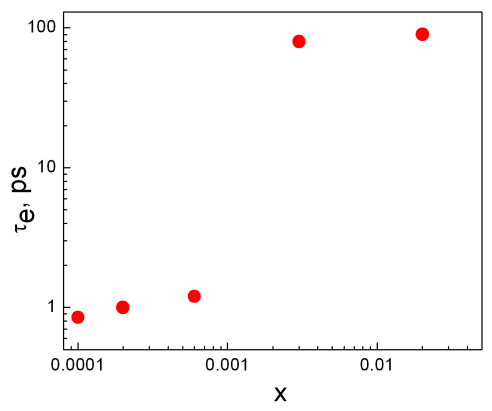

Fig. 2. Electron lifetime dependence on the Mn content in $\mathrm{Ga}_{1-x} \mathrm{Mn}_{x} \mathrm{As}$.

The dependence of the electron lifetime on the Mn content at the room temperature is shown in Fig. 2. The values of this parameter comparable to 1 ps 
are, most probably, determined by the electron capture at $\mathrm{As}_{\mathrm{Ga}}$ centers. Electrons are captured by the single-ionized arsenic antisites [3], thus the $\mathrm{Mn}^{++}$acceptors should tend to increase the rate of this capture - an effect which is opposite to our experimental observations. It is interesting to note that structural defects in the same set of $\mathrm{Ga}_{1-x} \mathrm{Mn}_{x}$ As samples as the ones used in our work were studied in [6] by measuring the lattice constant of the layers. For Mn contents smaller than $0.3 \%$ an anomalous decrease in the lattice constant was observed, which was attributed to the change of the charge state of $\mathrm{As}_{\mathrm{Ga}}$ defects.

Both the increasing electron lifetime and the decreasing lattice constant could be explained if we assume that Mn-doping causes the reduction of $\mathrm{As}_{\mathrm{Ga}}$ defect density in the layers. It is known that these defects can relatively easy undergo the transition $\left(\mathrm{As}_{\mathrm{Ga}} \rightarrow \mathrm{As}_{\mathrm{i}}+\mathrm{V}_{\mathrm{Ga}}\right)$ to the metastable state [7]. This transition causes a large lattice relaxation and the reduction of the number of electrically active defects acting as the electron traps. Because during the low temperature growth both As and Mn atoms are competing for the same Ga sites, it is possible that this competition results in the decreasing number of As $\mathrm{sa}_{\mathrm{Ga}}$ defects and in the increase in the electron lifetime. Moreover, because substitutional Mn causes a much smaller lattice distortion than As antisites [6], the replacement of $\mathrm{As}_{\mathrm{Ga}}$ by $\mathrm{Mn}_{\mathrm{Ga}}$ will result in an initial decrease in the lattice constant at dilute Mn contents.

In conclusion, we have used the optical pump - terahertz probe technique for measuring electron lifetime in $\mathrm{Ga}_{1-x} \mathrm{Mn}_{x}$ As epitaxial layers with various $\mathrm{Mn}$ contents with the subpicosecond temporal resolution. A strong increase in the lifetime from the values typical of LTG GaAs to the values comparable with the lifetimes in standard semi-insulating GaAs substrates was evidenced for the layers with more than $0.3 \% \mathrm{Mn}$. As a probable cause of this effect we propose the reduction of As-antisite traps due to the Mn incorporation.

\section{References}

[1] T. Jungwirth, K.Y. Wang, J. Mašek, K.W. Edmonds, Jürgen König, Jairo Sinova, M. Polini, N.A. Goncharuk, A.H. MacDonald, M. Sawicki, A.W. Rushforth, R.P. Campion, L.X. Zhao, C.T. Foxon, B.L. Gallagher, Phys. Rev. B 72, 165204 (2005).

[2] T. Dietl, Semicond. Sci. Technol. 17, 377 (2002).

[3] A. Krotkus, J.L. Coutaz, Semicond. Sci. Technol. 20, S142 (2005).

[4] S. Kim, E. Oh, J.U. Lee, D.S. Kim, S. Lee, J.K. Furdyna, Appl. Phys. Lett. 88, 262101 (2006).

[5] J. Sadowski, J.Z. Domagała, J. Bak-Misiuk, S. Kolesnik, M. Sawicki, K. Swiatek, J. Kanski, L. Ilver, V. Strom, J. Vac. Sci. Technol. B 18, 1697 (2000).

[6] J. Sadowski, J.Z. Domagala, Phys. Rev. B 69, 075206 (2004).

[7] M. Kaminska, Phys. Scr. T19B, 551 (1987). 